\title{
Globe
}

Revue internationale d'études québécoises

\section{Sexe, médias et... hypermoralisation}

\section{Maria Nengeh Mensah}

Volume 12, numéro 2, 2009

Images et représentations de la sexualité au Québec

URI : https://id.erudit.org/iderudit/1000713ar

DOI : https://doi.org/10.7202/1000713ar

Aller au sommaire du numéro

Éditeur(s)

Globe, Revue internationale d'études québécoises

ISSN

1481-5869 (imprimé)

1923-8231 (numérique)

Découvrir la revue

Citer cet article

Mensah, M. N. (2009). Sexe, médias et... hypermoralisation. Globe, 12(2), 169-180. https://doi.org/10.7202/1000713ar d'utilisation que vous pouvez consulter en ligne.

https://apropos.erudit.org/fr/usagers/politique-dutilisation/ 


\section{PERSPECTIVE}

\section{SEXE, MÉDIAS ET... HYPERMORALISATION}

MARIA NENGEH MENSAH

Université du Québec à Montréał

Attendons de voir ce que les petites filles en chandail bedaine vont devenir! On s'en reparlera dans 10 ans. Elles ne deviendront pas forcément des femmes soumises et aliénées. Et elles ne reviendront pas nécessairement à des postures pré-féministes'!

Nathalie COLLARD

Les pages qui suivent constituent une critique du discours moralisateur qui circule largement au Québec en ce moment à propos du sexe, des jeunes et des médias. Lélément déclencheur, pour moi, comme féministe, a été un rapport du Conseil du statut de la femme: Le sexe dans les médias: obstacle aux rapports égalitaires ${ }^{2}$. Il faut voir là un cas de figure des diatribes de chercheurs, universitaires, militants et autres acteurs, dont plusieurs féministes et médias aussi, qui intellectualisent et dynamisent le mouvement. Ce qui me trouble principalement concerne une sorte de

$$
+4
$$

1. Nathalie COLlarD, citée dans Monique DURAND et Lisa Marie NoEl, "Hypersexualisation des filles. Echec du féminisme?", Gazette des femmes, vol. 27, n², septembre-octobre 2005, p. 16.

2. Ginette Plamondon, Annie Desaulniers et Nathalie RoY, Le sexe dans les médias: obstacles aux rapports égalitaires, Québec, Conseil du statut de la femme, 2008. 
panique morale que je ne m'explique pas, car elle s'impose sournoisement comme une évidence, insoupçonnée: le sexe public est mauvais, les médias sont méchants et il est de la responsabilité de tous (de l'État, de l'industrie médiatique, du personnel éducatif et des parents) - sauf des jeunes visés - de rétablir l'ordre moral de l'égalité sexuelle. Il est temps de troubler ces lieux communs qui se posent comme critiques et libérateurs alors qu'ils constituent le redéploiement du dispositif de sexualité, pour reprendre le concept développé par Michel Foucault.

\section{LE SEXE DÉRANGE}

L'été dernier, le Conseil du statut de la femme a donc émis un nouvel avis intitule Le sexe dans les médias: obstacle aux rapports égalitaires ${ }^{3}$. Ce document d'une centaine de pages présente les effets néfastes de la "sexualisation de l'espace public ${ }^{4}$ " sur les jeunes. Le Conseil veut démontrer que la tendance à représenter les femmes comme des objets sexuels à des fins strictement commerciales est un phénomène inquiétant, d'autant plus inquiétant qu'il compromettrait les rapports sociaux égalitaires entre les hommes et les femmes, une des valeurs fondamentales de la société québécoise:

Outre la diffusion croissante de contenus sexuels dans les médias et l'invasion de ceux-ci dans la vie des jeunes, c'est en raison de la vision stéréotypée des rapports hommes-femmes qu'ils véhiculent et du caractère crucial, pour la socialisation, de l'âge adolescent, que nous tenons à alerter les autorités compétentes 5 .

Le Conseil ne s'en tient toutefois pas à sonner l'alarme. Les auteures s'emploient non seulement à recommander au gouvernement de resserrer les règles du jeu pour les producteurs de médias, diffuseurs et publicitaires, ce qui est de bonne guerre, mais aussi à préciser quelles normes sexuelles devraient orienter l'éducation à la sexualité en milieu scolaire afin de promouvoir l'égalité, de développer un esprit critique et de responsabiliser les acteurs. La chercheuse principale, Ginette Plamondon, aurait choisi d'éviter le terme "hypersexualisation" dans le titre du document, car le préfixe hyper, accolé au mot sexualisation, suggérerait "le dépassement d'une norme que le Conseil aurait bien été en peine de définir ${ }^{6}{ }$. C'est pourtant bien ce

$$
+4
$$

3. Ibid.

4. Ibid., p. 3.

5. Ibid.

6. Paule BELLEAU, "Responsabilicés partagées", Gazette des femmes, vol. 30 , $n^{\circ} 2$, septembre-octobre 2008 , p. 11. 
dont il est question dans cet avis: l'établissement d'une nouvelle règle sexuelle hypermorale sous le couvert de la promotion des idéaux de la sexualité égalitaire entre les hommes et les femmes. C'est à cet égard qu'un glissement a lieu de la promotion d'une sexualité dite égalitaire à celle d'une sexualité normale, marquée au sceau du féminisme.

Plusieurs féministes arrivent à cette position car elles ont fait l'épreuve de ce que c'est que d'être traitée comme un objet de façon inconvenante, que ce soit à travers des commentaires désobligeants dans la rue, le harcèlement en milieu de travail ou l'agression sexuelle. Une réaction possible consiste à exiger de ne pas être traitée comme un objet sexuel. Cette réaction féministe est nécessaire. N'était de celle-ci, nous n'aurions pas aujourd'hui des centres d'aide pour les victimes d'agression sexuelle ni de maisons d'hébergement pour les femmes battues. Mais il faut aussi voir que la sexualité n'est pas seulement un monde de risques et de misère, c'est aussi un monde inouï de désirs, d'épanouissement et d'affirmation. Ce que le féminisme nous a montré par ailleurs, c'est qu'il faut faire une place au plaisir ${ }^{7}$. Ainsi, à partir de la notion du droit au plaisir sexuel, les femmes ont revendiqué les droits de refuser le mariage, de maîtriser leur fécondité et de vivre leur sexualité hors des carcans hétéronormatifs. La dissociation de la sexualité et de la reproduction a été un puissant levier de notre émancipation érotique. Cependant, suivant la même logique, la constitution de cette sphère de la sexualité-plaisir reste étroitement associée à un impératif social.

En fait, il s'agit d'un dérapage de ce que Michel Foucault a nommé le "dispositif de sexualité". Car lorsqu'il est question de sexe, un ensemble d'investissements sociaux construisent la sexualité comme le centre des discours contemporains. Ces

contrôles sociaux [...] entrepren[nent] de protéger, de séparer, de prévenir, signalant partout des périls, éveillant des attentions, appelant des diagnostics, entassant des rapports, organisant des thérapeutiques; autour du sexe, ils irradient les discours, intensifiant la conscience d'un danger incessant qui relance à son tour l'incitation à en parler ${ }^{8}$.

$$
+4
$$

7. "Le féminisme doit contribuer à accroître le plaisir et la joie des fermmes, et non seulement à réduire nos malheurs" ["Feminism must increase women's pleasure and joy, not just decrease our misery"] (Carol S. VANCE, "Pleasure and Danger: Towards a Politics of Sexuality", dans Pleasure and Danger: Exploring Female Sexuality, 2 édition, New York, Pandora, 2002 [1992], p. 24).

8. Michel FOUCAULT, Histoire de la sexwalité. Tome I: La volonté de savoir, Paris, Gallimard, 1976, p. 43. 
Foucault définit ce "dispositif" comme un ensemble hétérogène du dit aussi bien que du non-dit, comportant des discours, des institutions, des aménagements architecturaux, des décisions réglementaires, des lois et des politiques; mais également des énoncés scientifiques ainsi que des propositions philosophiques et des morales. Lorsqu'il est question du sexe, le dispositif de sexualité favorise l'instauration d'une méfiance à l'égard de ce qu'on appelait jadis les déviances: l'exhibitionnisme, le voyeurisme, la sexualité de groupe, l'homosexualité, la prostitution, etc. En effet, il semble que plus on s'éloigne de "l'honnête coït entre adultes consentants", pour reprendre la formule de l'Antimanuel d'éducation sexuelle ${ }^{9}$, plus il faut être sûr que le désir qui nous y porte est "éclairé", surtout s'il se trouve que l'on est âgé de 12 à 18 ans, que l'on soit une fille ou un garçon. Ce dispositif de sexualité dont parle Michel Foucault a cela de particulier qu'il permet de normaliser la sexualité de chacun en tant qu' "enjeu de vérité " et "savoir du sujet ${ }^{10}$ ".

Partant du point de vue que la sexualité se doit d'être dépourvue de stéréotypes sexuels (comme si cela était possible, ou même souhaitable ${ }^{11}$ ), le Conseil se réfere à des définitions retenues tantôt par le ministère de l'Éducation du Québec ${ }^{12}$, tantôt par la Quatrième Conférence mondiale sur les femmes ${ }^{13}$, afin d'intégrer le droit à l'égalité entre les femmes et les hommes, et d'en arriver à adopter l'expression de "sexualité égalitaire». Mais le Conseil se fonde surtout sur l'analyse exhaustive de ses propres publications, en regroupant des caractéristiques biologiques, psychologiques et sociales constituant "l'idéal de sexualité égalitaire que le Conseil a développé au fil des années [et] qui l'a guidé dans ses réflexions ${ }^{14}{ }^{\prime} . \mathrm{Si}$, pour le Conseil, "la sexualisation de l'espace public réfere à l'omniprésence de la sexualité dans l'espace collectif qu'est l'espace public" et si «la sexualité traditionnellement considérée du ressort de la vie privée constitue maintenant un élément important de la vie publique ${ }^{15}$ ", il est faux de croire que ceci est un phénomène nouveau. Le dispositif de sexualité, selon Foucault, serait apparu

$$
+\div
$$

9. Marcela LACUB et Parrice MANiglier, Antimanuel d'éducation sexuelle, Rosny, Bréal, 2005.

10. Michel FouCAULT, op. cit., p. 76 et 93.

11. Voir Richard PARKER et Peter AGgleton (dir.), Culture, Society and Sexuality. A Reader, $2^{c}$ édition, New York, Routledge, 2007.

12. Francine DUQUET, L'éducation à la sexualité dans le contexte de la refforme de l'éducation, Québec, ministère de l'Éducarion, 2003, p. 9.

13. ORGANISATION DES NATIONS UNIES, Déclaration de Beijing. Rapport de la Quatrième Conférence mondiale sur les femmes (Beijing, 4-15 septembre 1995), New York, Nations Unies, 1995.

14. Ginette Plamondon, Annie Desaulniers ec Nathalie Roy, op. cit., p. 24.

15. Ibid., p. 17. 
à l'époque moderne, alors qu'un seul type de sexualité entre adultes consentants devenait la norme, et aurait été suivi d'une explosion du discours sur la sexualité, participant au contrôle des pratiques sexuelles. "Ce projet d'une "mise en discours" du sexe, il s'était formé, il y a bien longtemps, dans une tradition ascétique et monastique ${ }^{16}{ } \ldots$ et il semble encore bien vivant aujourd'hui.

\section{OMNIPRÉSENCE DE LA MORALE SUBSTANTIALISTE}

Les auteures du Conseil condamnent l'exposition aux images pornographiques (définies ici comme sexistes) sous prétexte qu'elles imposeraient, elles, des normes sexuelles et des modèles de relations amoureuses aux jeunes, particulièrement vulnérables, "en construction identitaire ${ }^{17}$ ". Les jeunes font l'objet d'attentions spéciales, puisqu'on pense qu'il faut leur laisser le temps de découvrir leur sexualité. Pourtant, cette découverte est déjà profondément minée par le spectre de l'hypermoralisation... cette tendance renouvelée de la morale sexuelle des siècles derniers, substantialiste, qui consiste à vouloir démontrer que le sexe est mauvais et dangereux.

Insistons sur le préfixe hyper, car on n'aura jamais autant entendu parler de la "sexualisation précoce des fillettes ${ }^{18}$ " ou encore de la "pornographisation de la société ${ }^{19}$ ", et l'usage de ces expressions témoigne à lui seul de l'importance de cette sensibilité excessive aux représentations de la sexualitéplaisir dans la sphère publique et, parallèlement, d'une mise en discours de plus en plus moralisatrice. Or, paradoxalement, c'est au moyen d'une lutte à l'hypersexualisation de l'espace public (dont le Conseil fait éminemment partie) que ledit Conseil prétend protéger l'authenticité du désir sexuel, le mettre à l'abri de toutes les pressions et de toutes les dominations, et le soumettre ainsi à un seul régime du "comment vivre" sa sexualité.

D'un point de vue philosophique, il existe, selon Marcela Iacub, deux grands types de morales sexuelles ${ }^{20}$. Les premières, dites «substantialistes", imposent des critères communs de ce qui est bon pour chacun dans le domaine sexuel, en dépit du point de vue que les individus eux-

$$
+++
$$

16. Michel Foucault, op. cit., p. 29.

17. Ginette Plamondon, Annie Desaulniers et Nathalie Roy, op. cit., p. 11.

18. Pierrette BOUCHARD, Nathalie BOUCHARD et Isabelle BOILY, La sexualisation précoce des filles. Essais, Montréal, Sysiphe, 2005.

19. Richard POULIN, en collaboration avec Mélanie CLAUDE, *La mode hypersexualisée s'inspire de la pornographie ", http://sisyphe.org/imprimer.php3?id_article=3074 (15 décembre 2008).

20. Marcela LACUB et Patrice Maniglier, op. cit., p. 280-290. 
mêmes peuvent avoir sur ce qui leur convient. Au nom d'une certaine idée de la vertu, ce modèle autoritaire nous dit en substance: "Je ferai ton bien en dépit de toi et même contre toi». En revanche, le second type de morales sexuelles, "consensualistes", cherche plutôt à définir un cadre qui garantisse à chacun le droit de vivre sa sexualité selon ses propres valeurs. Or, je crois que la panique morale actuelle concernant l'hypersexualisation de l'espace public, notamment celle des jeunes, montre qu'on n'a aucunement réussi à rompre avec une morale substantialiste.

Au Québec, du XVIII ${ }^{e}$ siècle jusque dans la première moitié du $\mathrm{XX}^{e}$ siècle, le clergé catholique avait pour mission de faire respecter le commandement de Dieu dictant la fonction maritale et hétérosexuelle de "l'œuvre de la chair ${ }^{21} "$. Bien que nous ayons l'impression en tant que société moderne d'être passés au-delà de l'ancienne morale, entre autres grâce à la révolution sexuelle et au mouvement féministe, une sorte de sacralité entourant la sexualité demeure. Les dogmes judéo-chrétiens hanteraient-ils toujours le Québec?

En effet, le sexe apparaît toujours comme un noyau d'authenticité qu'il faut préserver coûte que coûte: "Comme une sorte de petite boîte, de petit secret enfermé au plus profond de nous, qu'il faut laisser s'avouer progressivement à travers notre vie elle-mêmen. Iacub poursuit :

Ce qui importe par-dessus tout, ce n'est pas que nous puissions « vivre notre sexualité", c'est plutôt que cet ensemble secret d'aspirations et de fantasmes, si profondément liés à ce que nous sommes, reste intouché, sacré, au sens étymologique du terme, c'est-à-dire séparé2 ${ }^{22}$.

L'espace public doit donc être débarrassé du sexe, car le sexe, qui doit rester en deçà de toute relation de pouvoir, risquerait d'en être contaminé. Il importe par-dessus tout de préserver la pureté de la sexualité. Cette volonté est particulièrement évidente à travers l'inquiétude face au sexe rendu public et accessible aux jeunes par le biais des médias. Nous revenons encore et toujours à l'idée selon laquelle il existerait de "bons" moyens d'exprimer sa sexualité et d'autres qui seraient culturellement, moralement et politiquement répréhensibles. Peu importe le consentement des jeunes ou leurs désirs. Importe davantage notre désir de pureté, d'égalité, notre conscience féministe revenant immanquablement à la charge. Bien entendu, ce n'est pas

$$
+4
$$

21. Voir Éric VOLANT, "Éthique», Joseph J. LÉvY et André DUPRAS (dir.), Questions de sexualité au Québec, Montréal, Liber, 2008, p. 115-121.

22. Marcela LACUB er Parrice MANIGLIER, op. ctt. p. 286. 
le Conseil du statut de la femme à lui seul qui veut faire de "la" sexualité égalitaire une valeur, en prétendant la reconnaître institutionnellement et fonder sur elle un certain nombre de restrictions, de délimitations et d'interdictions. Des chercheurs universitaires intellectualisent aussi l'hypermoralisation, sans oublier que les médias eux-mêmes ont fait couler beaucoup d'encre à son sujet ${ }^{23}$.

\section{LÀ OU IL Y A SEXUALITÊ, IL Y A POUVOIR}

Que les médias véhiculent la conception d'une sexualité basée sur des rapports inégalitaires entre les femmes et les hommes doit être dénoncé. J'en conviens. Je ne suis pas contre l'idée de critiquer les contenus sexistes ou misogynes, et même racistes, homophobes, transphobes et "putophobes ${ }^{24}$ " de plusieurs médias consommés par les jeunes et les moins jeunes. Loin de moi aussi l'idée de remettre en question l'incitation croissante à dire le sexe et à montrer la sexualité publiquement, à la mettre à nu, littéralement, qui existe depuis déjà plus de trois siècles, le XXI siècle n'étant qu'une suite logique de ce dispositif de la sexualité mis en cuvre avec les espoirs de la modernité. Par contre, il semble naîf de croire que la sexualité égalitaire est extérieure aux relations de pouvoir, et que le sexe peut être complètement dénué de stéréotypes, de domination ou de rapports de force. Même lorsqu'elle est vécue ou représentée - ce qui n'est pas la même chose ${ }^{25}$ - de façon égalitaire, la sexualité s'insère dans le même cadre que tout autre rapport social et, en cela, ne peut être soustraite aux rapports de force. Qui plus est, ces rapports sont susceptibles de se produire partout, et surtout là où on les attend le moins.

L'essai de la journaliste Pascale Navarro Pour en finir avec la modestie féminine $e^{26}$ en dit long là-dessus, mais il n'a malheureusement pas été cité par le Conseil. Navarro dépeint le phénomène de l'hypersexualisation

$$
+\div
$$

23. Du côté des médias de masse québécois, la presse écrite a publiê en 2008 plus d'une trentaine d'articles dont le titre contenaic le mot "hypersexualisation". Des médias spécialisés, comme la revue électronique A Babord! et la Gazette des femmes, ont également proposé des dossiers thématiques sur le sujet au couranc de la mème année.

24. La putophobie est une des discriminations les plus partagées dans notre culture, mais à ce jour elle demeure peu documentée. Le manifeste de MAITTRESSE NIKITA et Thierry SCHAFAUSER, Fières d'être putes (Montreuil-sous-bois, Éditions L'Alciplano, 2007), explique : «Il y a plusieurs logiques qui déterminent le sentiment putophobe. Les deux principales sont de désigner les putes comme: des victimes, trop connes, incapables de savoir ce qui est bon pour elles [...]; des délinquantes, vecteurs de désordre et d'épidémie [...]. Dans les deux cas, les putophobes agissent en fonction d'un senciment de supérioritén (p. 24).

25. En effer, un problème du discours hypermoralisateur réside parfois dans sa cendance à confondre les deux. On ne distingue plus entre la représentation du sexe à laquelle les jeunes sonc confrontés et leurs praciques sexuelles.

26. Pascale NAVARRO, Pour en finir avec la modestie féminine, Montréal, Boréal, 2002. 
comme une stratégie discursive ayant pour but de restreindre l'avancée des femmes et des filles dans l'espace public, empêchement jusqu'ici secondé par l'idée d'une modestie féminine. Car la réalité, c'est que les filles et les femmes sont maintenant présentes partout au Québec, dans les rues, sur le marché du travail, en politique, dans les facultés universitaires, et elles y réussissent très bien. La modestie, elle, "consiste en une série de comportements qui "définissent" la féminité. Est féminine celle qui se montre délicate, réservée, douce, discrète. Surtout : elle sait passer en second et s'effacer derrière les hommes (son mari, son frère, son patron, etc.) ${ }^{27}$ ". Le visage de la modestie aurait changé depuis les années 1980 : les filles et les adolescentes cherchent non plus à être de bonnes filles, mais plutôt à être belles et sexy. L'objet des préoccupations dans les deux cas est le même: se conformer au modèle de ce que l'on attend d'elles. Les modèles de féminité sont construits et peuvent être déconstruits, selon Navarro, mais l'angoisse de déplaire continue d'être entretenue par une éducation moralisatrice et une culture de la modestie ${ }^{28}$.

Malheureusement, la réflexion qui abonde en ce moment n'aborde que trop rarement les rapports de force, de négociation et de résistance exercés par les filles face à la victimisation et par rapport à la sexualisation. Si le contenu des médias a été cerné, peu de recherches ont porté sur la réception des messages médiatisés par les différents publics au Québec, ce qui permettrait de mieux évaluer, selon Joseph Lévy, «leurs retombées sur le développement des identités sexuelles et des scénarios relationnels et sexuel ${ }^{29}$ ". On a plutôt tendance, comme l'a fait le Conseil, à aborder ces questions en faisant valoir de quelle manière les filles et les femmes assimilent, à travers l'industrie culturelle et les médias, des modèles de relation de couple systématiquement hétérosexuels, souvent empreints de violence, d'agressivité, de jalousie et de contrôle excessif.

\section{LES JEUNES SANS DISCERNEMENT}

Je ne reviendrai pas ici sur les écueils méthodologiques de la position féministe des conseillères du "statut de la femme " dans les médias québécois. Cette tâche revient aux scientifiques qui étudient la question des comportements sexuels, et qui sont mieux placés pour nous rappeler qu'il existe autant d'études qui démontrent qu'il n'y a pas d'effets là où les autres en

$$
+*+
$$

27. Ibid., p. 17.

28. Ibid., p. 51.

29. Joseph J. LEVY, "Médias", Joseph J. LÉVY et André DUPRAS (dir.), Questions de sexualité au Québec, Montréal, Liber, 2008, p. 315. 
avaient repérés. Par ailleurs, il m'apparaît flagrant que le Conseil décrit abondamment les jeunes, le public-cible du sexe dans les médias, comme s'ils formaient une masse inerte, unique, sans discernement et dépourvue de sens critique lorsque vient le temps de naviguer sur Internet, de recevoir une publicité ou de regarder la télévision. Or, le Conseil cite, en note de bas de page, une étude non publiée qu'il a menée auprès des jeunes et qui révèle que ceux-ci sont critiques par rapport aux médias et à la pornographie. Mais ces études ne semblent pas avoir été intégrées dans le corps du texte (voir notamment la page 41 de l'Avis). Ainsi, la présidente du Conseil, Christine Pelchat, soutenait dans un communiqué que:

L'adolescence est une phase de construction identitaire, une période de changements et c'est à ce stade que les rapports sociaux de sexe prennent forme chez l'individu, ce qui le rend particulièrement réceptif aux modèles ambiants. Devant l'influence des médias et des modèles sexuels stéréotypés qui y sont propagés, c'est la construction de l'identité sexuelle des adultes de demain qui est en jeu ${ }^{30}$.

Tout le monde, à différents moments de sa vie, est susceptible d'être plus vulnérable aux messages qui lui sont présentés. Certes. Mais cette vulnérabilité s'applique autant à la voisine de cinquante ans récemment divorcée qu'aux hommes ayant des relations sexuelles avec d'autres hommes et qui ne s'identifient pas comme gais. Surtout, le problème avec cette définition, c'est qu'elle ne permet pas de tenir compte de la capacité d'esprit critique de l'audience jeunesse.

Rappelons que tout ceci dépend inévitablement de la théorie des médias que l'on va choisir. Et puisque les ancrages théoriques du Conseil semblent être liés à une vision psychosociologique d'influence marxiste selon laquelle les médias provoqueraient des "déceptions massives" qui ont un effet de "narcotisation ${ }^{31}$ ", il n'est pas surprenant de l'entendre tirer la sonnette d'alarme concernant la menace des médias à contenus sexuels. Les travaux de théoriciens d'après la Deuxième Guerre mondiale allaient dans le même sens alors qu'ils craignaient la réapparition du fascisme ${ }^{32}$. Le postulat derrière ce $++$

30. CONSEIL DU STATUT DE LA FEMME DU QUÉBEC, Communiqué. Le sexe dans les médias: obstacle aux rapports égalitaires, http://sisyphe.org/spip.php?article3005 (15 décembre 2008).

31. Voir Paul F. LAZARSfeld et Robert K. MERTON, "Mass Communication, Popular Taste, and Organized Social Action ", Lyman BRYSON (dir.), The Communication of Ideas : a Series of Addresses, New York, Insticute for Religious and Social Srudies, 1948, p. 95-118.

32. Voir Theodor ADORNO et Max HORKHEIMER, "The Culture Industry: Enlightenment as Mass Deception ", dans Dialectic of Enlightenment [Dialektik der Aufklärung], New York, Continuum, 1993 [1944], p. 94-136. 
modèle de l'influence des médias, toutefois, est que le sens du message est établi uniquement par l'émetteur et que le processus de réception est déterminé par le contenu prédéfini du message. Pourtant (et nous-mêmes le vivons à tous les jours dans plusieurs contextes différents), il n'en est jamais ainsi puisque nous sommes actifs dans la réception des messages. On pourrait même avancer que les contenus sexuels sont constitués comme tels justement à travers le travail de décodage et de resignification que font les lecteurs et les lectrices pour les lire, les voir, les entendre ${ }^{33}$. Pourquoi les jeunes ne seraientils pas aussi actifs?

Et si l'on s'appuyait sur une vision plus dynamique de la communication médiatisée, qu'elle mette en scène la sexualité-plaisir ou non, afin d'imaginer les jeunes comme des êtres capables d'interpréter le sexe dans les médias de différentes façons, et de concevoir que ceci a peu à voir avec les comportements sexuels qu'ils ont ou qu'ils auront? Bon nombre de spécialistes des médias qui étudient la culture ont justement examiné l'usage des messages médiatisés par les individus, passant du registre d'utilisateur conciliant à celui de critique, résistant ou contestataire ${ }^{34}$. Cette posture ne chercherait plus à montrer un lien direct entre le traitement médiatique de la sexualité ou des rapports sociaux de sexe ${ }^{35}$ et le comportement sexuel des garçons et des filles. L'enquête tenterait plutôt de saisir les significations multiples et les interprétations inattendues ou subversives (oui, il y en a) du discours dominant sur le sexe dans l'espace public. Les jeunes qui négocient les messages véhiculés seraient ainsi considérés comme ayant un rôle et un pouvoir dynamiques. Des sujets actifs et producteurs de changement social, et non plus des masses inertes qui subissent le discours d'autrui.

$$
++
$$

33. Maria Nengeh MENSAH et Thomas HAIG, "Becoming Visible? Being Heard? Community Interpretations of Visibility and Disclosure in First-person Accounts of People Living with HIV/AIDS in Quebec Media", 5' Conférence internationale AIDS in Culture, Mexico, 11 décembre 2008.

34. Mentionnons des travaux fondareurs: Stuart HALL, "Encoding/Decoding", Culture, Media, Language: Working Papers in Cultural Studies, 1972-79, Londres, Hutchinson, 1980, p. 128-138; Ien ANG, Watching Dallas: Soap Opera and the Melodramatic Imagination, Londres, Mechuen, 1985 ; David MORlEY, Television, Audiences and Cultural Studies, Londres, Routledge, 1992; Constance PENLEY, "Feminism, Psychoanalysis and the Study of Popular Culture", Lawrence Grossberg, Cary NeLSON et Paula A. Treichler (dir.), Cultural Studies, Londres, Routledge, 1992, p. 479-494.

35. L'expression "rapports sociaux de sexen est habituellement utilisée pour référer à ce que les féministes anglophones nomment gender; elle renvoie à l'assignation socioculturelle des individus selon une certaine compréhension des catégories de sexe en termes de féminité et de masculinité. 


\section{MIEUX "PÉDAGOGISER"}

L'avis du Conseil du statut de la femme nous laisse avec l'impression que la socialisation des filles est en danger. L'hypermoralisation a pour tâche de prohiber les manifestations visibles de la sexualité, le sexe dans l'espace public, et de semer une panique morale à l'égard des produits culturels accessibles aux jeunes. Tout se passe comme s'il nous était impossible de différencier - dans la pratique, à tout le moins - le genre et la sexualité; bien que les deux soient inextricablement liés, nous avons tendance à accoler à l'identité "femme" une compréhension de la sexualité incluant un ensemble de dispositions paradoxales, de hiérarchies d'appartenances et d'exclusions. Or c'est là que se situe un des plus grands défis actuels du féminisme. Selon Chantal Nadeau, il importe "de faire et de penser l'engagement comme une mouvance critique par laquelle les tensions qui émanent du genre et de la sexualité se nourrissent en fait de leurs désirs contradictoires ${ }^{36}{ }^{\text {. }}$. Est-ce qu'il est réellement possible d'isoler une seule "cause" aux problèmes de dévalorisation et d'estime de soi qui affectent les jeunes? Il existe bien des jeunes qui consomment des images sexuelles dans les médias sans pour autant avoir "une image génitale et irréaliste de la sexualité» ou encore des filles qui n'ont pas nécessairement "des préoccupations obsessives par rapport à leur $\operatorname{corps}^{37}$ ". De plus, les jeunes sont soumis à toutes sortes d'influences très diverses et nous ne pouvons mesurer le poids de chacune.

Aux États-Unis, les programmes d'éducation sexuelle de plusieurs États sont basés sur la promotion de l'abstinence, soutenus par les incitatifs financiers de l'administration Bush aux écoles qui choisissent de faire la promotion de la chasteté avant le mariage ${ }^{38}$. Cette insistance à l'égard de l'abstinence sexuelle aurait tendance à faire de la sexualité un risque pour la santé des jeunes, un élément à surveiller de près. Au Québec, le contexte semble certes différent. Mais l'est-il vraiment? Depuis 2005, le ministère de l'Éducation a retiré graduellement le cours de formation personnelle et sociale de la grille-matières à l'école secondaire, de sorte que l'éducation à la sexualité ne relève désormais plus d'une seule matière ou d'une personne en particulier. L'éducation sexuelle s'inscrit dorénavant dans l'ordre des affaires publiques: du domaine général de formation à la santé et du bien-être

$$
+\div
$$

36. Chantal NADEAU, "L'urgence-désir comme engagement", Canadian Journal of Communication, vol. 31, n'4, 2006, p. 921.

37. Ginette Plamondon, Annie Desaulniers et Nathalie RoY, op. cit., p. 62.

38. John S. SANTELLI et al., "Abstinence and Abstinence-Only Education: a Review of US Policies and Programs", Journal of Adolescent Health, vol. 38, n 1, 2006, p. 72-81. 
relevant de la responsabilité d'un ensemble de partenaires. Le sexe est devenu immanquablement public. Plutôt que de remettre en question cette véritable entreprise de salut, le Conseil s'inquiète que l'on néglige, dans les éléments prescriptifs de l'éducation sexuelie, "la mise en valeur de l'estime de soi, le respect de soi et de l'autre, la vie affective et amoureuse et les relations égalitaires ${ }^{39}$ ". Il propose donc de mieux encadrer la sexualité des jeunes - ce que Foucault nomme la "pédagogisation du sexe de l'enfant". Du coup, l'avis du Conseil confirme une fois de plus que le sexe des jeunes est beau mais dangereux, et qu'il demeure un enjeu important autour duquel d'innombrables dispositifs institutionnels et stratégies discursives sont aménagés. Comme le voulait la stratégie répressive à l'époque victorienne face à l'onanisme, la méthode actuelle consiste toujours à entourer, diriger, protéger, défendre, même, le sexe de l'enfant. Cette tactique du discours, il faudrait la "considérer comme un réseau productif qui passe à travers tout le corps social beaucoup plus que comme une instance négative qui a pour fonction de réprimer ${ }^{40}$ ".

Voilà comment on passe à côté de l'instabilité des identités de genre, des dynamiques de consommation de contenus sexuels dans les médias et d'un questionnement direct des pratiques sexuelles dans leurs rapports aux stéréotypes et à la norme. Voilà comment, à travers l'instauration actuelle d'une panique morale ancienne, le dispositif de la sexualité reprend toutes ses forces. Enfin, voilà comment, "en attendant de voir ce que les petites filles en chandail bedaine vont devenir ", dire que le sexe dans l'espace public est mauvais ou dangereux parle de nous, nous marque, nous arrête. À quand un envers aux discours moralisateurs?

$$
+4
$$

39. Ginetre Plamondon, Annie Desaulniers et Nathalie Roy, op. cit., p. 84.

40. Michel FOUCAULT, op. cit., p. 149. 